\title{
A singular minimizer of a smooth strongly convex functional in three dimensions
}

\author{
Vladimír Šverák Xiaodong Yan
}

\section{Introduction}

We consider variational integrals of the form,

$$
I(u)=\int_{\Omega} f(D u(x)) d x,
$$

where $\Omega \subset \mathbf{R}^{n}$ is a bounded open set, $u: \Omega \rightarrow \mathbf{R}^{m}$ is a mapping belonging to $W^{1,2}(\Omega), D u(x)$ denotes the gradient matrix of $u$ at $x \in \Omega$, and $f$ is a smooth strongly convex function with uniformly bounded second derivatives defined on the set $M^{m \times n}$ of all real $m \times n$ matrices. We recall that $f$ is said to be strongly convex if there exists a constant $\nu>0$, such that for all

$\xi \in M^{m \times n}, X \in M^{m \times n}$, the inequality $f_{p_{\alpha}^{i} p_{\beta}^{j}}(X) \xi_{\alpha}^{i} \xi_{\beta}^{j} \geq \nu|\xi|^{2}$ holds. Here and in what follows we will be using Einstein's summation convention.

We shall consider the regularity of minimizers of $I$ in $W^{1,2}(\Omega)$. Here by a minimizer we mean a function $u \in W^{1,2}(\Omega)$ such that for any smooth function $\phi: \Omega \rightarrow \mathbf{R}^{m}$ compactly supported in $\Omega$ the inequality $I(u+\phi) \geq I(u)$ holds. When $f$ is strongly convex, it is not difficult to see that $u$ is a minimizer of $I$ if and only if $u$ is a weak solution of the Euler - Lagrange equation of $I$, i.e. $u$ satisfies (in the sense of distributions)

$$
\partial_{\alpha} f_{p_{\alpha}^{i}}(D u(x))=0, \quad i=1, \cdots m .
$$

A classical result of C.B. Morrey ([Mo]) says that when $n=2, m \geq 1$, every minimizer of $I(u)$ is regular. This is also the case when $n \geq 2, m=1$ by celebrated results of De Giorgi( [De1]) and Nash ([Na]). The methods used in the proof of De Giorgi and Nash can not be extended to the case $m \geq 2$ as shown by a counterexample of De Giorgi ([De2]). The first example of a nonsmooth minimizer for a smooth strongly convex functional of the type (1.1) was constructed by Nečas in high dimensions (see $[\mathrm{Ne}]$ ). He considered the function $u: \mathbf{R}^{n} \rightarrow \mathbf{R}^{n^{2}}$ defined by

$$
u_{i j}=\frac{x_{i} x_{j}}{|x|}
$$


and for large $n$ constructed a strongly convex function $f$ on $M^{n \times n^{2}}$ for which $u$ is a minimizer of the corresponding functional $I$. Later Nečas, Hao and Leonardi $([\mathrm{HLN}])$ were able to modify this construction and make it work for $n \geq 5$. They used $u$ given by

$$
u_{i j}=\frac{x_{i} x_{j}}{|x|}-\frac{|x|}{n} \delta_{i j}
$$

Important counterexamples to regularity of solutions of elliptic systems which are not of the form (1.2) can be found in [GM] and [NJS]. For a comprehensive treatment of regularity questions we refer the reader to [Gi]. Interesting sufficient conditions for regularity are given in [Ko].

The purpose of this paper is to give a counterexample to regularity of weak solutions of (1.2) in the case $n=3, m=5$. We use exactly the same $u$ defined by (1.4) and construct a smooth strongly convex function $f$ such that $u$ is a minimizer of $I$. The main idea of our construction is the following. Let $K=\{\nabla u(x), x \in \Omega\}$ be the set of gradients of $u$. We find a null Lagrangian $L$ (see Definition 2.1 below) such that

$$
\nabla L(X)=\nabla f(X), \quad \forall X \in K
$$

for a smooth strongly convex function $f$. Then $u$ will satisfy the Euler Lagrange equation of $I$ automatically. To find the null Lagrangian we use the symmetries of the function $u$. We will see below that there is, up to a multiplicative factor, a unique quadratic null Lagrangian on $M^{5 \times 3}$ which is invariant under the symmetries of the function $u$. It turns out that this null Lagrangian satisfies a necessary and sufficient condition for the existence of a strongly convex $f$ satisfying (1.5).

\section{Preliminaries}

First we introduce some basic facts about null Lagrangians.

Definition 2.1 (see [Ba1]) $L: M^{m \times n} \rightarrow \mathbf{R}$ is a null Lagrangian if for each smooth $u: \mathbf{R}^{n} \rightarrow \mathbf{R}^{m}$,

$$
\operatorname{div} \nabla L(\nabla u(x))=0 .
$$

We recall the following classical theorem about null Lagrangians (see [Da] or $[\mathrm{BCO}])$.

Proposition 1 Let $L: M^{m \times n} \rightarrow \mathbf{R}$, the following conditions are equivalent:

i) $L$ is a null Lagrangian.

ii) $L$ is a linear combination of subdeterminants.

iii) $L$ is rank-one affine, i.e. $t \rightarrow L(A+t B)$ is affine for each $A \in M^{m \times n}$ and each $B \in M^{m \times n}$ with rank $B=1$. 
From now on, let $\Omega$ be the unit ball in $\mathbf{R}^{3}$. Consider $u=\left(u_{i j}(x)\right)$ given by

$$
u_{i j}(x)=\frac{x_{i} x_{j}}{|x|}-\frac{|x|}{3} \delta_{i j}, \quad i, j=1, \ldots, 3
$$

Then for each $x \in \Omega, u(x) \in\left\{A \in M^{3 \times 3}, A=A^{t}, \operatorname{tr} A=0\right\} \cong \mathbf{R}^{5}$. For each $R \in S O(3)$ we have

$$
u(R x)=R u(x) R^{t}=\rho_{5}(R) u(x)
$$

where we denote by $\rho_{2 i+1}$ the unique irreducible representation of $S O(3)$ of dimension $2 i+1$. This notation will be used throughout the paper. An easy calculation shows that

$$
\nabla u(R x)=\rho_{5}(R) \nabla u(x) R^{t}=\rho_{5} \otimes \rho_{3}(R) \nabla u(x) .
$$

Lemma 2.1 There exists a unique (up to multiplication by a scalar) quadratic invariant null Lagrangian $L$ on $M^{5 \times 3}$ which is invariant under the above action of $S O(3)$.

Proof Consider the tensor space $T=\left\{a_{i j k} \in\left(\mathbf{R}^{3}\right)^{\otimes 3} \mid a_{i j k}=a_{j i k}, a_{i i k}=0\right\}$. Clearly we have $T \cong \mathbf{R}^{15} \cong M^{5 \times 3}$. By the Clebsch-Gordan formula (see $[\mathrm{BD}]$ ), we know that

$$
\rho_{5} \otimes \rho_{3}=\rho_{7} \oplus \rho_{5} \oplus \rho_{3}
$$

We now identify the quadratic null Lagrangians on $M^{5 \times 3}$ with $\Lambda^{2} \mathbf{R}^{3} \otimes \Lambda^{2} \mathbf{R}^{5} \cong$ $\operatorname{Hom}\left(\Lambda^{2} \mathbf{R}^{3}, \Lambda^{2} \mathbf{R}^{5}\right)$ and consider the representation $\sigma$ of $S O(3)$ on $\operatorname{Hom}\left(\Lambda^{2} \mathbf{R}^{3}\right.$, $\Lambda^{2} \mathbf{R}^{5}$ ) induced by $\rho_{3} \otimes \rho_{5}$. By classical group representation theory (see [BD]) we have

$$
\sigma=\rho_{9} \oplus \rho_{7} \oplus \rho_{5} \oplus \rho_{5} \oplus \rho_{3} \oplus \rho_{1}
$$

Therefore we see there is a unique one dimensional invariant subspace.

\section{Constructions}

\subsection{Construction of $L$}

Now we calculate explicitly the invariant quadratic null Lagrangian which will be denoted by $L$ in what follows. (We slightly abuse the notation, since $L$ is only determined up to a multiplicative factor.) Since we have $M^{5 \times 3}=$ $V_{7} \oplus V_{5} \oplus V_{3}$, where $V_{i}$ is the $i$-dimensional irreducible invariant subspace. 
We know from the classical invariant theory (see [We1]) that $L$ must be of the following form:

$$
L(A)=\alpha|X|^{2}+\beta|Y|^{2}+\gamma|Z|^{2}
$$

where $A \in M^{5 \times 3}, \quad A=X+Y+Z, \quad$ with $X \in V_{7}, Y \in V_{5}, Z \in V_{3}$.

We identify $M^{5 \times 3}$ with $T=\left\{a_{i j k} \in\left(\mathbf{R}^{3}\right)^{\otimes 3} \mid a_{i j k}=a_{j i k}, a_{i i k}=0\right\}$ in the obvious way. Now we use a classical procedure to decompose $T$ into irreducible subspaces ( see [We1]). We first decompose $T$ into the trace-free part $T^{\prime}$ and its orthogonal supplement $T_{3}$, i.e. $T=T^{\prime} \oplus T_{3}$. An easy calculation shows that the projection on $T_{3}$ is given by $a_{i j k} \rightarrow-\frac{1}{5} \delta_{i j} \eta_{k}+\frac{3}{10} \delta_{k i} \eta_{j}+\frac{3}{10} \delta_{j k} \eta_{i}$ with $\eta_{k}=a_{k i i}, k=1,2,3$. Then we decompose $T^{\prime}$ by using symmetrizations. We have $T^{\prime}=T_{1} \oplus T_{2}$, where the projection on $T_{1}$ is given by symmetrization, i.e. $\quad a_{i j k} \rightarrow \frac{1}{3}\left(a_{i j k}+a_{j k i}+a_{k i j}\right)$; the projection on $T_{2}$ is given by $a_{i j k} \rightarrow$ $\frac{1}{3}\left(a_{i j k}+a_{j i k}-a_{k j i}-a_{k i j}\right)$, which corresponds to the following Young tableau:

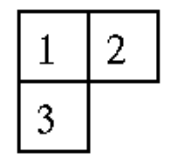

We remark that the antisymmetric part of any tensor in $T$ is 0 . We now identify $T_{1}$ with $V_{7}, T_{2}$ with $V_{5}, T_{3}$ with $V_{3}$.

We now use the condition that $L$ has to vanish on rank-one matrices. These matrices correspond to the tensors in $T$ which are of the form $a_{i j k}=c_{i j} \xi_{k}$, where $C=\left(c_{i j}\right)$ is a trace-free symmetric matrix. A direct calculation of the norms of the projections $a_{i j k}^{l}$ of $a_{i j k}$ to $T_{l}$ gives

$$
a_{i j k}=a_{i j k}^{1}+a_{i j k}^{2}+a_{i j k}^{3}
$$

with

$\left|a_{i j k}^{1}\right|^{2}=\frac{1}{3}|C|^{2}|\xi|^{2}+\frac{2}{5}|C \xi|^{2}, \quad\left|a_{i j k}^{2}\right|^{2}=\frac{2}{3}|C|^{2}|\xi|^{2}-|C \xi|^{2}, \quad\left|a_{i j k}^{3}\right|^{2}=\frac{3}{5}|C \xi|^{2}$.

From this we see that, using the same notation as above, $L(A)=\alpha|X|^{2}+$ $\beta|Y|^{2}+\gamma|Z|^{2}$ vanishes on rank one matrices if and only if

$$
\alpha: \beta: \gamma=(-2): 1: 3
$$

For our purpose, we will take $\alpha=-2, \beta=1, \gamma=3$ in the following. 


\subsection{The construction of $f$}

We recall that $K=\{\nabla u(x), x \in \Omega\}=\left\{\nabla u(x), x \in S^{2}\right\} \subset M^{5 \times 3}$, where $u$ is defined by (1.4), and where we have identified the $3 \times 3$ trace-free symmetric matrices with $\mathbf{R}^{5}$. A necessary condition for the existence of a strongly convex function $f$ satisfying (1.5) is that there exist $\delta_{0}>0$, such that

$$
\nabla L(X) \cdot(Y-X) \leq-\delta_{0}|Y-X|^{2} \quad \forall X, Y \in K
$$

We will see this condition is satisfied.

Lemma 3.1 For any $X=\nabla u(x), Y=\nabla u(y) \in K$, where $x, y \in S^{2}$, we have

$$
L(\nabla u(x)-\nabla u(y)) \geq 8|x-y|^{2} .
$$

Proof: First we note that we have the following decomposition for $\nabla u(x) \in$ $K, x \in S^{2}$.

$$
u_{i j k}=u_{i j k}^{1}+u_{i j k}^{2}+u_{i j k}^{3}
$$

where

$$
\begin{aligned}
u_{i j k}^{1} & =-x_{i} x_{j} x_{k}+\frac{1}{5}\left(x_{i} \delta_{j k}+x_{j} \delta_{i k}+x_{k} \delta_{i j}\right), \\
u_{i j k}^{2} & =0 \\
u_{i j k}^{3} & =\frac{4}{5}\left(x_{i} \delta_{j k}+x_{j} \delta_{i k}-\frac{2}{3} x_{k} \delta_{i j}\right) .
\end{aligned}
$$

and

$$
\left|u_{i j k}^{1}\right|^{2}=\frac{2}{5}, \quad\left|u_{i j k}^{3}\right|^{2}=\frac{64}{15} .
$$

Hence $\quad L(\nabla u(x)) \equiv 12 \quad \forall x \in S^{2}$.

Since $L$ is quadratic, we have

$$
L(\nabla u(x)-\nabla u(y))=2 L(\nabla u(x))-2 L(\nabla u(x), \nabla u(y)),
$$

where we slightly abuse the notation by using $L$ also for the symmetric bilinear form corresponding to the quadratic form $L$.

$$
\begin{aligned}
L(\nabla u(x), \nabla u(y))= & -2 u_{i j k}^{1}(x) \cdot u_{i j k}^{1}(y)+3 u_{i j k}^{3}(x) \cdot u_{i j k}^{3}(y) \\
= & -2\left(-x_{i} x_{j} x_{k}+\frac{1}{5}\left(x_{i} \delta_{j k}+x_{j} \delta_{k i}+x_{k} \delta_{i j}\right)\right) \cdot \\
& \left(-y_{i} y_{j} y_{k}+\frac{1}{5}\left(y_{i} \delta_{j k}+y_{j} \delta_{i k}+y_{k} \delta_{i j}\right)\right) \\
& +3\left(\frac{4}{5}\right)^{2}\left(x_{i} \delta_{j k}+x_{j} \delta_{k i}-\frac{2}{3} x_{k} \delta_{i j}\right) \cdot\left(y_{i} \delta_{j k}+y_{j} \delta_{i k}-\frac{2}{3} y_{k} \delta_{i j}\right) \\
= & -2\langle x, y\rangle^{3}+14\langle x, y\rangle .
\end{aligned}
$$


Let $t=\langle x, y\rangle$. Then $-1 \leq t \leq 1$, and we have

$$
\begin{aligned}
L(\nabla u(x)-\nabla u(y)) & =2 L(\nabla u(x))-2 L(\nabla u(x), \nabla u(y)) \\
& =2(1-t)\left(-2\left(1+t+t^{2}\right)+14\right) \\
& \geq 16(1-t) \\
& =8|x-y|^{2} .
\end{aligned}
$$

The proof of Lemma 3.1 is finished.

We have $L(X)=12$ for all $X \in K$ and therefore Lemma 3.1 gives

$$
\begin{aligned}
\nabla L(\nabla u(x)) \cdot(\nabla u(y)-\nabla u(x))= & -L(\nabla u(x)-\nabla u(y)) \\
& +L(\nabla u(x))+L(\nabla u(y))-2 L(\nabla u(x)) \\
= & -L(\nabla u(x)-\nabla u(y)) \\
\leq & -8|x-y|^{2} .
\end{aligned}
$$

Since we have

$$
\frac{21}{4}|x-y|^{2} \leq|X-Y|^{2} \leq \frac{20}{3}|x-y|^{2}
$$

for $X=\nabla u(x), Y=\nabla u(y)$, we see that the condition (3.1) is satisfied.

It turns out that (3.1) together with the fact that $L$ is constant on $K$ is also sufficient for the existence of a strongly convex function satisfying (1.5). A natural attempt to make such an extension would be to take the convex hull of $K$ and consider a modification of the corresponding Minkowski functional. However, since the convex hull of $K$ may not be smooth at $K$, we need to slightly modify this construction.

We fix $\epsilon>0$ (the exact value will be specified later) and for each $X \in K$, consider the 10 dimensional ball of radius $r_{\epsilon}=\epsilon|\nabla L(X)|=\epsilon \sqrt{160}$ passing through $X$ centered at $X^{\prime}=X-\nabla L(X) \epsilon$. We will denote the ball as $B_{X^{\prime}, r_{\epsilon}}$.

Lemma 3.2 When $\epsilon$ is sufficiently small we have

$$
\nabla L(X)(\tilde{Y}-X) \leq-\frac{1}{2}|\tilde{Y}-X|^{2}
$$

for each $X \in K$ and each $\tilde{Y} \in B_{Y^{\prime}, r_{\epsilon}}$, where $B_{Y^{\prime}, r_{\epsilon}}$ is defined above, with $Y$ being an arbitrary point of $K$.

Proof: The inequality

$$
\left|\tilde{Y}-Y^{\prime}\right|^{2} \leq \epsilon^{2}|\nabla L(Y)|^{2}
$$

gives

$$
\nabla L(Y) \cdot(\tilde{Y}-Y) \leq-\frac{1}{2 \epsilon}|\tilde{Y}-Y|^{2}
$$


Hence

$$
\begin{aligned}
\nabla L(X) \cdot(\tilde{Y}-X)= & (\nabla L(X)-\nabla L(Y)) \cdot(\tilde{Y}-Y)+\nabla L(Y) \cdot(\tilde{Y}-Y) \\
& +\nabla L(X) \cdot(Y-X) \\
\leq & 10|Y-X||\tilde{Y}-Y|-\frac{1}{2 \epsilon}|\tilde{Y}-Y|^{2}-\frac{6}{5}|Y-X|^{2}
\end{aligned}
$$

and the statement follows easily.

Let $S=\cup_{X \in K} B_{X^{\prime}, r_{\epsilon}}$. When $\epsilon$ is small, the boundary of $S$ is smooth by elementary results about tubular neighborhoods (see [Hi] or [We2]). Lemma 3.2 implies that (for sufficiently small $\epsilon$ ) all the eigenvalues of the second fundamental form of $\partial S$ are negative and bounded above uniformly on $K$ by a negative constant $\gamma$ ( i.e the principle curvatures $k_{i}(X) \leq \gamma<0, \forall i$ and $\forall X \in K)$. Since $\partial S$ is smooth, we conclude that $\partial S$ is locally strongly convex at any point of $U \cap \partial S$, where $U$ is a small neighborhood of $K$.

Now take $G$ to be the convex hull of $S$ in $V_{7} \oplus V_{3}$. Using Lemma 3.2 and the fact that $\partial S$ is smooth and locally strongly convex in $U \cap \partial S$, we infer that $U \cap \partial G=U \cap \partial S$ when the neighborhood $U$ of $K$ is choosen to be sufficiently small. Let

$$
F_{1}(X)=\min \{t \geq 0, X \in t G\}, \quad F(X)=12 F_{1}^{2}(X) .
$$

Then $F$ is smooth and strongly convex in $U$ (see [Ro]), and $\nabla L(X)=\nabla F(X)$ for each $X \in K$. Let $\phi$ be a smooth non-negative mollifier with support in $B_{1}$ and let

$$
F_{\delta}=\phi_{\delta} * F
$$

where $\phi_{\delta}(x)=\delta^{-n} \phi\left(\frac{x}{\delta}\right)$. Define

$$
H_{\delta, \tau}(X)=F_{\delta}+\tau|X|^{2}
$$

Let $0 \leq \eta \leq 1$ be a smooth cut-off function satisfying $\eta=1$ in $U^{\prime}$, and $\eta=0$ outside $U$, where $U^{\prime}$ is an open neighborhood of $K$ satisfying $\bar{U}^{\prime} \subset U$.

Now define

$$
H=(1-\eta) H_{\delta, \tau}+\eta F
$$

A straightforward calculation shows that $H$ is a strongly convex function on $V_{7} \oplus V_{3}$ when $\delta$ and $\tau$ is small enough. Now take $f(A)=H(X+Y)+|Z|^{2}$ to be our final function, where $A \in M^{5 \times 3}, \quad A=X+Y+Z$, with $X \in V_{7}, Y \in$ $V_{5}, Z \in V_{3}$. We know that $f$ coincide with $F(X+Y)+|Z|^{2}$ in the neighborhood of $K$, thus $\nabla L(X)=\nabla f(X)$ for all $X \in K$ holds and $f$ is a smooth strongly convex function everywhere. This proves the following theorem: 
Theorem 1 Let $\Omega=\left\{x \in \mathbf{R}^{3},|x|<1\right\}$ and let $u: \Omega \rightarrow \mathbf{R}^{5}$ be defined by $u_{i j}=\frac{x_{i} x_{j}}{|x|}-\frac{|x|}{3} \delta_{i j}, i, j=1, \cdots 3$, where we identify the $3 \times 3$ symmetric tracefree matrices with $\mathbf{R}^{5}$. Then $u$ is a minimizer of $I(u)=\int_{\Omega} f(D u(x))$, where $f$ is the smooth strongly convex function defined above.

\section{References}

[Ba1] J. M. Ball: Convexity conditions and existence theorems in nonlinear elasticity. Arch. Rational Mech. Anal. 63(1978), 337-403.

[BCO] J. M. Ball, J. C. Currie, P. J. Olver: Null lagrangians, weak continuity, and variational problems of arbitrary order, J. Funct. Anal. 41(1981) 135174.

[BD] Theodor Bröcker, Tammo tom Dieck: Representations of Compact Lie Groups, Springer-Verlag, New York, 1985

[Da] B. Dacorogna: Direct methods in the Calculus of Variations, SpringerVerlag, 1989.

[De1] E. De Giorgi: Sulla differenziabilità e l'analitità della estremali degli integrali multipi regolari, Mem. Accad. Sci. Torino cl. Sci. Fis. Mat. Nat.(3) 3(1957), 25-43.

[De2] E. De Giorgi: Un esempio di estremali discontinue per un problema variazionale di tipo ellittico, Boll. UMI 4(1968), 135-137.

[Gi] M. Giaquinta: Multiple integrals in the calculus of variations and nonlinear elliptic systems, Princeton University Press, Princeton, 1983.

[GM] E. Giusti, M. Miranda: Un esempio di soluzione discontinua per un problem di minimo relativo ad un integrale regolare del calcolo delle varazioni, Boll. Un. Mat. Ital. 2 (1968), 1-8.

[Hi] Morris W. Hirsch: Differential Topology. New York, Springer Verlag, 1976.

[HLN] W. Hao, S. Leonardi, J. Nečas: An example of irregular solution to a nonlinear Euler-Lagrange elliptic system with real analytic coefficients. Ann. Scuola Norm. Sup. Pisa Cl. Sci. (4) 23 (1996), no. 1, 57-67.

[Ko] A. I. Koshelev: Regularity problem for quasilinear elliptic and parabolic systems, Springer, 1995. 
[Mo] C. B. Morrey: Multiple integrals in the calculus of variations . Springer Verlag, Heidelberg, New York, (1966).

[Na] J. Nash: Continuity of solutions of parabolic and elliptic equations, Amer. J. Math. 8(1958), 931-954.

[Ne] J. Nečas: Example of an irregular solution to a nonlinear elliptic system with analytic coefficients and conditions of regularity, Theory of Non Linear Operators, Abhandlungen Akad, der Wissen. der DDR(1977), Proc. of a Summer School held in Berlin (1975).

[NJS] J. Nečas, O. John, J. Stará: Counterexample to the regularity of weak solutions of elliptic systems. Comment. Math. Univ. Carolinae 21 (1980), 145-154.

[Ro] Tyrrell R. Rockafellar: Covex analysis. Princeton, N.J. Princeton University Press, 1970.

[We1] H. Weyl: The Classical Groups. Their Invariants and Representations. Princeton, New Jersey. 1939

[We2] H. Weyl: On the volume of tubes. American Journal of Mathematics, 61(1939) 461-472. 